\title{
Estudo de um modelo de Laboratório EAD (LabEAD) aplicado na Engenharia da Computação
}

\author{
Ricardo de Andrade ${ }^{14}$ (ricardo.andrade@ufabc.edu.br) \\ (https://orcid.org/0000-0002-5812-3754) \\ Marcelo Nogueira Tirolli ${ }^{13}$ (marcelo.tirolli@cursos.univesp.br) \\ (https://orcid.org/0000-0002-3465-9642) \\ Lucas Pires Gomes de Oliveira ${ }^{13}$ (lucas.p.oliveira@unesp.br) \\ (https://orcid.org/0000-0001-5576-927X) \\ Edilon de Oliveira França ${ }^{13}$ (edilon.franca@ @ursos.univesp.br) \\ (https://orcid.org/0000-0002-4040-7409) \\ Danyela Cardoso Carvalho ${ }^{12}$ (danyelacardoso@usp.br) \\ (https://orcid.org/ 0000-0002-5284-1050) \\ Édison Trombeta de Oliveira ${ }^{12}$ (edison.trombeta@univesp.br) \\ (https://orcid.org//0000-0001-9935-4260)
}

${ }^{1}$ Departamento de Pós-graduação Lato Sensu em EAD, Universidade Virtual do Estado de São Paulo (UNIVESP), São Paulo, Brasil

${ }^{2}$ Departamento de Pós-graduação Stricto Sensu, Universidade de

São Paulo (USP), São Paulo, Brasil

${ }^{3}$ Departamento de Pós-graduação Stricto Sensu, Universidade do Estado de São Paulo (UNESP), São Paulo, Brasil

${ }^{4}$ Departamento de Pós-graduação Stricto Sensu, Engenharia da Informação,

Universidade Federal do ABC (UFABC), Santo André/SP, Brasil

Resumo: Práticas em laboratórios são essenciais no ensino de engenharia, uma vez que permitem que estudantes aprendam de forma eficaz e desenvolvam habilidades indispensáveis para o seu futuro como profissionais na área. As práticas possuem uma série de fatores, tais como custos e infraestrutura que dificultam a sua disponibilização. Entretanto, laboratórios remotos tem grandes potenciais para possibilitar essas práticas laboratoriais de maneira eficiente e com custos reduzidos quando comparado aos laboratórios tradicionais. Atualmente percebe-se o crescimento na implementação dessas soluções em diversas áreas de ensino e profissionais, nas quais faz necessário conhecimentos práticos principalmente devido à pandemia de COVID-19. Este artigo investigou a implementação de laboratório remoto, ferramenta que possibilita a realização de ensino prático visando ao estudo de caso e aplicação de um modelo de validação de roteiros laboratoriais nas disciplinas de eletrônica, a fim de preparar melhor os alunos da UNIVESP para o mercado de trabalho, auxiliar o trabalho dos professores e facilitadores em relação ao entendimento de um circuito eletrônico. Os resultados desta pesquisa foram satisfatórios e os autores recomendam a implementação de um estudo deste projeto nos polos da UNIVESP e/ou de outras universidades que atuem à distância.

Palavras-chave: LabEAD, Acesso Remoto, Circuitos Eletrônicos, Laboratório Remoto, LabEAD UNIVESP.

\section{Study of an e-Learning Laboratory Model (LabEAD) applied in Computer Engineering}

Abstract: Laboratory practices are essential in engineering education, as they allow students to learn effectively and develop indispensable skills for their future as professionals in the field. The practices have a series of factors, such as costs and 
infrastructure, that make their availability difficult. However, remote laboratories have great potential to enable these laboratory practices in an efficient and cost-effective way when compared to traditional laboratories. Currently, we can see the growth in the implementation of these solutions in several areas of education and professionals, in which practical knowledge is necessary, mainly due to the COVID-19 pandemic. This paper investigated the implementation of a remote laboratory, a tool that enables practical teaching aimed at the case study and application of a validation model of laboratory scripts in electronics disciplines, in order to better prepare UNIVESP's students for the market of job and to assist the work of professors and tutors in relation to the understanding of an electronic circuit. The results of this research were satisfactory and the authors recommend the implementation of a study of this project in the centers of UNIVESP and/or other universities that work with e-learning.

Keywords: LabEAD, Remote Access, Electronic Circuits, Remote Laboratory, LabEAD UNIVESP.

\section{Introdução}

Há muito tempo se discute a educação a distância no Brasil e como lidar com ela por meio da utilização da tecnologia (TORI, 2010). A realização de experimentos práticos é um aspecto importante durante a aprendizagem de ciências pelos alunos, sendo uma atividade indispensável no ensino de engenharia, por exemplo. Como alternativas aos laboratórios presenciais há os laboratórios com acesso remoto e simuladores (GUIMARÃES et al.,2013).

O tema deste artigo surgiu a partir da observação da ausência de práticas de laboratório em disciplinas de eletrônica ofertadas nos cursos de Engenharia da Computação e Ciência da Computação pela Universidade Virtual do Estado de São Paulo (UNIVESP). Sabe-se que um terço da grade curricular de um curso de Engenharia da Computação é voltado às disciplinas de eletrônicas analógica, digital e microcontroladores. Essas disciplinas são tratadas $100 \%$ em teorias e no máximo há simulação de circuitos, que não fazem parte da grade obrigatória e nem de avaliação, sendo apenas referenciada como conteúdo extracurricular. Devido a esse gap, elaborou-se um estudo de caso a respeito da implantação de um modelo de laboratório de acesso remoto, LabEAD, que é abordado em diversas literaturas existentes (GUTIÉRREZ et al., 2017; HERADIO et.al., 2016) principalmente durante a pandemia do COVID-19 (HAYASHI, 2020; RICCARDO et al.; 2021; RACZYŃSKI, 2021). Com base neste estudo, a melhor plataforma a ser utilizada neste projeto baseou-se no desenvolvimento de outros pesquisadores (HAYASHI,2020). Este ambiente permite que seja feita uma conexão remota em um laboratório para validar os resultados obtidos em cálculos teóricos, simulados e práticos .

As simulações virtuais permitem ao aluno executar programas construídos nas mais diversas linguagens em seu computador HTML5, C, JAVA, PYTHON, MATLAB etc. (HAYASHI, 2020). Mesmo com metodologias particulares da educação a distância, o entendimento de conceitos básicos pode não ser um substituto aos dos trabalhos em laboratórios presenciais, uma vez que os modelos construídos são uma aproximação da realidade (HAYASHI, 2020). Há inúmeras literaturas que discorrem a respeito dessa modalidade de ensino. Essas serão exploradas na fundamentação teórica.

Neste projeto são investigados gaps e possíveis soluções para o apoio às disciplinas práticas de eletrônica em instituição de ensino superior visando principalmente o distanciamento social e a aprendizagem a distância. Por meio dos estudos realizados em literaturas, surgiu a oportunidade de pesquisa em laboratório à distância para apoiar disciplinas de Graduação de Engenharia na UNIVESP e, futuramente, demais instituições de ensino no Brasil. 


\section{Fundamentação teórica}

Há alguns autores (DEBOER, 2019) que são ligados à área de pesquisa em ensino de ciências que já mostraram que a realização de experiências práticas em laboratórios presenciais de ensino representa um fator importante no ciclo de aprendizagem dos alunos. Conforme demonstraram esses estudos, um ensino experimental inapropriado pode provocar desmotivações com claro efeito negativo no rendimento e aproveitamento dos alunos (GUIMARÃES et al., 2013). Entretanto, construir laboratórios experimentais presenciais em todas as escolas e universidades do Brasil é algo que dificilmente irá se concretizar um dia, pois esses laboratórios normalmente exigem investimentos relativamente altos, com a compra de equipamentos, custos de manutenção e de pessoal especializado na área de ensino. Como alternativa viável de contornar esse problema, a implantação de laboratórios de acesso remoto junto aos laboratórios de simulações virtuais pode propiciar uma concreta alternativa para essa questão.

Foi proposto em 2012 um acesso remoto chamado de WebLab, o qual inicialmente serviria para a realização de experimentos em tempo real como apoio ao ensino público da disciplina de Física no Brasil, além disso houve a implementação com Redes de Petri Coloridas em um ambiente simulado (JUNIOR et al., 2012).

Os laboratórios de acesso remoto permitem que os alunos controlem equipamentos de um laboratório real, de forma que os experimentos ocorram em local distinto à sua manipulação (GUIMARÃES et al., 2013). Neste caso, o aluno deve ter acesso à Internet, e a abordagem permite que recursos possam ser compartilhados entre diversas instituições de ensino e pesquisa. Outras vantagens deste projeto, são a redução do custo da execução dos experimentos por aluno e a maior disponibilidade do laboratório, que não fica limitado aos horários fixos de laboratório presencial (PEREIRA et al., 2018).

No mundo, existem outros estudos também relacionados aos laboratórios remotos. Por exemplo o laboratório VISIR (Virtual Systems in Reality) do Instituto de Tecnologia de Blekinge, o qual busca apoiar o ensino de circuitos elétricos e eletrônicos, e vêm sendo expandido em universidades de diversos países da Europa como Portugal, Espanha, Áustria, e na Ásia e América do Sul com participação da Índia, e Argentina, respectivamente (LIMA et.al., 2016).

As tecnologias virtuais têm o potencial de fazer com que os alunos se sintam mais comprometidos e motivados (GUTIÉRREZ et.al., 2017). Pesquisas nessas tecnologias abrem novos caminhos para o ensino e a aprendizagem já que existem inúmeros estudos de caso que investigam esta área ao usar tecnologias virtuais em ambientes educacionais, mas esses estudos tendem a se concentrar em experiências e tópicos específicos. Assim, é importante compreender designs instrucionais eficazes para uma melhor integração de da Realidade Virtual (virtual reality-VR) e Aumentada (augmented reality-AR) pois ainda não há uma visão clara de como integrar essas tecnologias de forma estável em um processo educacional.

No Brasil, o projeto VISIR+ suporta a instalação, configuração e uso de instâncias do VISIR. Resultados empíricos de dois anos sobre efeitos na aprendizagem relacionada à eletrônica analógica usando o VISIR estão presentes no trabalho de Garcia-Zubia (2016).

Houve muitas iniciativas além do VISIR no ramo de acesso remoto ao laboratório em diversos países. Sistemas como o LabEAD, RMCLab, por exemplo, foram projetos que visaram a construção de um osciloscópio remoto e a utilização das placas de FPGA, um dispositivo com acesso através de um navegador web à uma plataforma microcontrolada e monitorada por uma webcam que analisava o funcionamento de LED's durante experimentos de eletrônica (HAYASHI, 2020). 


\section{Metodologia}

O estudo realizado neste projeto utiliza o método descritivo de pesquisa sendo essencialmente quantitativo, com ênfase na observação e no estudo documental realizado por um teste de bancada e in loco para que haja acesso remoto dos alunos da UNIVESP das áreas de Engenharia e TI, e realizada a coleta de dados, sobre a percepção dos alunos com essa plataforma, os quais serão demonstrados no decorrer do projeto.

Neste projeto utilizou-se uma plataforma de LabEAD desenvolvida por alguns pesquisadores (HAYASHI,2020) que foi modificada para atender as necessidades desta pesquisa. As ferramentas de software utilizadas para construção do projeto LabEAD foram Fritzing, Arduino, Python, HTML/CSS e javascript. Também houve Interfaces humano-computador personalizadas, infraestrutura de comunicação em arquitetura cliente-servidor e protocolo serial para suporte ao experimento de eletrônica e coleta de dados. Através de estudos literários consistiu-se a possibilidade de adquirir uma série de conhecimentos que contribuíram para o objetivo proposto, envolvendo temas como:

- Estudos de viabilidade dentro do modelo de ensino da UNIVESP;

- Avaliação de aprendizagem dos alunos;

Após a fase anteriormente citada, foi estabelecido o cronograma conforme descrito as fases abaixo:

- Concepção e projeto;

- Implementação do roteiro;

- Implementação do hardware e software;

- Ensaios laboratoriais;

- Enquetes e coleta de dados dos alunos após a interação com os roteiros;

- Conclusão dos resultados obtidos.

A figura 1 mostra a arquitetura simplificada do LabEAD desenvolvida neste projeto. É possível observar que os alunos e professor acessam o laboratório remotamente (via software AnyDesk, por exemplo) e tem acesso aos experimentos que estão ligados em uma protoboard ou placa ilha ligados a um arduíno os quais são monitorados através de uma câmera acoplada e interface desenvolvida pelos autores desta pesquisa.

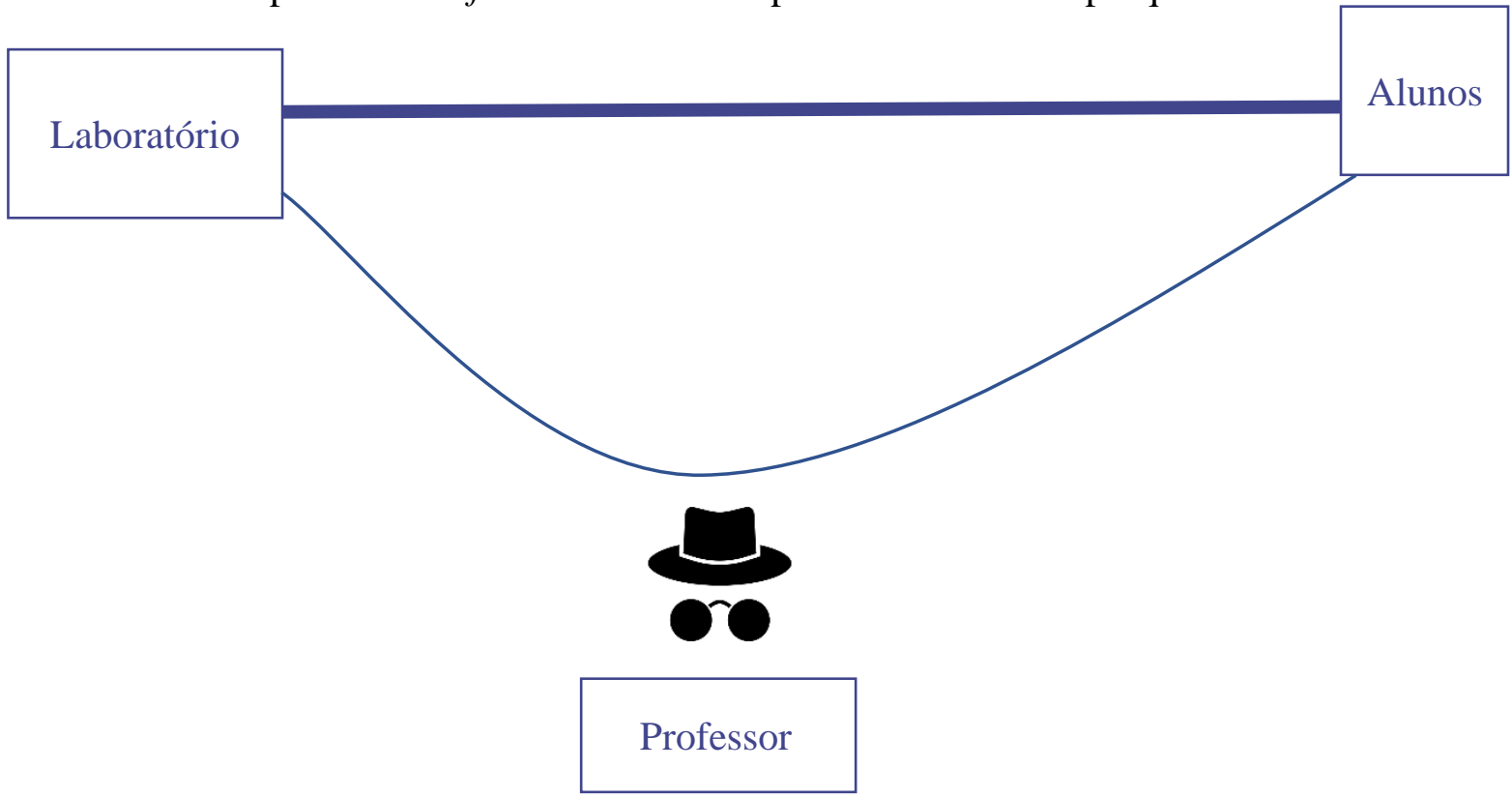

Figura 1 - Arquitetura do LabEAD. Fonte: Próprio autor. 
Quanto ao hardware, além de Arduino Mega, componentes eletrônicos foram utilizados, conforme Quadro 1.

Quadro 1 - Hardware utilizado (visão geral).

\begin{tabular}{|c|c|}
\hline Componentes & Equipamentos \\
\hline Fios para protoboard & Cabo USB \\
\hline Resistores de carbono de $1,1 \mathrm{~K} \Omega \stackrel{1 / 4 \mathrm{~W}}{ }$ & Placa padrão de circuito integrado \\
\hline Diodo Zener 1N4728 & Protoboard \\
\hline Estanho $40+60$ Chumbo & Arduíno Uno ou Arduíno Mega \\
\hline & Fonte de 9V e multímetro de bancada \\
\cline { 2 - 2 } & Ferro de solda \\
\hline
\end{tabular}

\section{Desenvolvimento}

Por meio do experimento representado na figura 2, onde se encontra um diodo Zener $1 \mathrm{~N} 4728$ e um resistor de $1,1 \mathrm{k} \Omega$. O aluno pode inserir o valor da tensão de $0 \mathrm{~V}$ até $10 \mathrm{~V}$, e lê-lo nos displays do arduíno e do multímetro de bancada. No exemplo apresentado na figura 2, é possível visualizar que o valor de entrada é $1,06 \mathrm{~V}$ e os de saída do arduíno e multímetro são $1,05 \mathrm{~V}$ e $1,04 \mathrm{~V}$, respectivamente. A figura 3 demonstra a montagem do circuito montado em protoboard utilizada neste projeto.

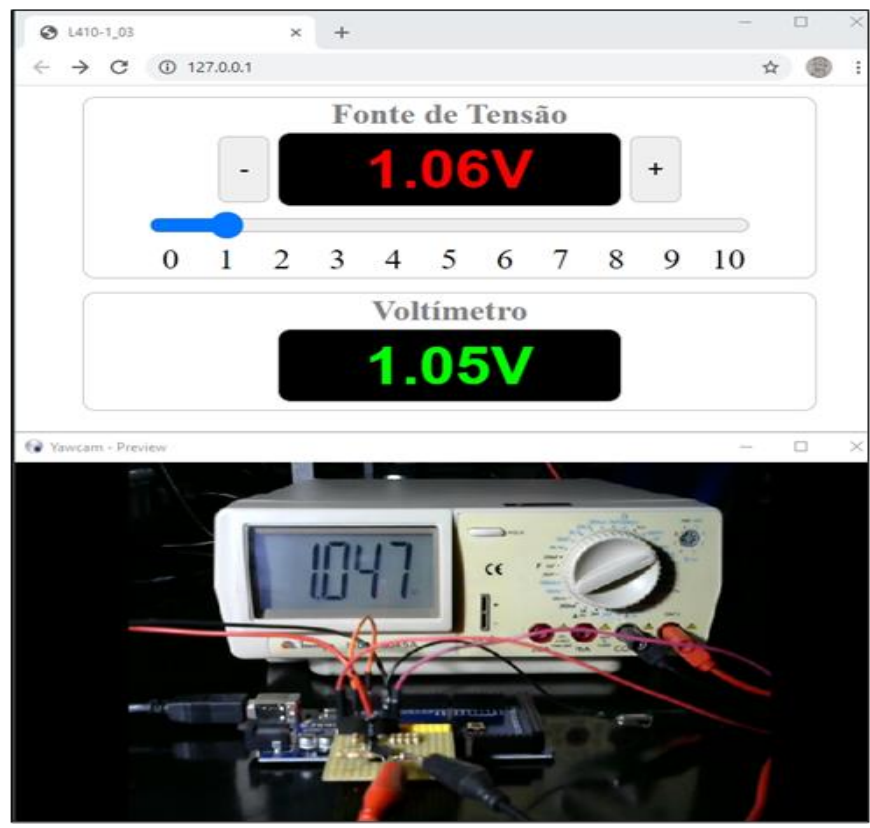

Figura 2 - Bancada com sistema LabEAD. Fonte: Próprio autor. 


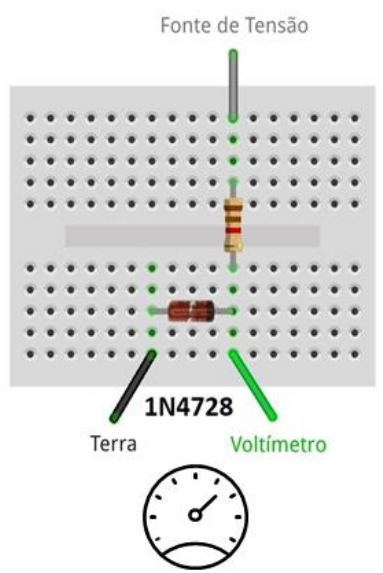

Figura 3 - Circuito montado em protoboard. Fonte: Próprio autor.

É possível observar que existe uma diferença de tensão apresentada entre o voltímetro do arduíno e o multímetro de bancada. Essa pequena diferença de 0,02V deve-se à tolerância de erro de cada equipamento, o que varia conforme o fabricante. Essa observação pode ser questionada aos alunos, obrigando-os a investigarem os datasheets das empresas fabricantes e incentivando-os assim a pesquisa e estudo dos equipamentos.

Conforme apresentado o experimento, seria possível a aplicação de uma avaliação dos conhecimentos dos alunos, por meio de um relatório do experimento prático apresentado pelo professor responsável pela disciplina ministrada, no caso de eletrônica analógica, podendo abranger em média 5 perguntas, por exemplo, a determinar o valor da tensão mostrada no multímetro do arduíno quando ajustada a fonte para $1.06 \mathrm{~V}$, explicar a curva do diodo Zener 1N4728, entre outras.

Nessa etapa do projeto, um breve vídeo descritivo do sistema LabEAD foi produzido e fornecido aos alunos de graduação da UNIVESP, junto a um formulário a ser respondido com base na percepção e entendimento individual do projeto. Esse questionário apresentado aos alunos foi composto por 11 questões, sendo 9 de múltipla escolha, 1 de dissertação e 1 em escala likert. As questões foram elaboradas visando o conhecimento dos alunos dos cursos de exatas da UNIVESP, abrangendo perguntas sobre idade, curso do aluno, sugestões, avaliação do projeto, entre outras.

\section{Resultados e discussões}

Após 60 dias de coleta de dados, os quais foram obtidos por meio das respostas dos alunos da UNIVESP a partir de um vídeo explicativo do projeto em questão, foram obtidas respostas de 23 discentes de graduação com as informações descritas nos gráficos a seguir. A figura 4, ilustra os gêneros dos entrevistados, estes dados possibilitam uma análise do público da instituição. E a figura 5, a faixa etária dos entrevistados:

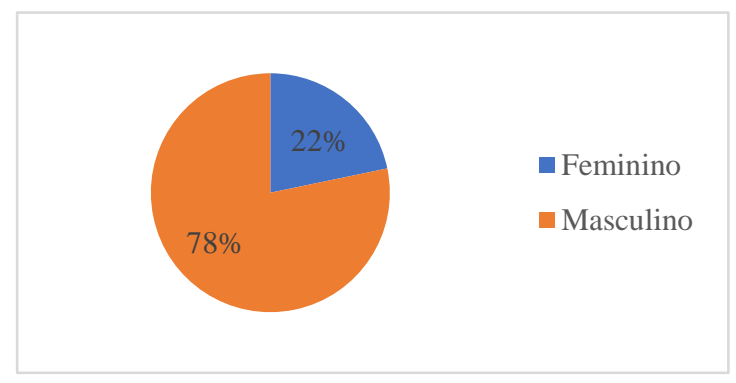

Figura 4 - Distribuição de gênero na UNIVESP. Fonte: Próprio autor. 


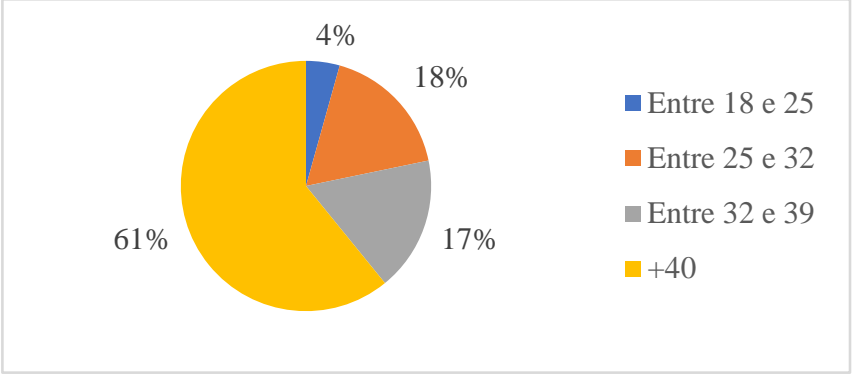

Figura 5 - Distribuição de idade. Fonte: Próprio autor.

As duas figuras anteriores (4 e 5) mostram que a maioria dos discentes são homens (78\%), e com $61 \%$ dos entrevistados tendo mais de 40 anos. Isso mostra uma desigualdade de gênero nos cursos de exatas de nível superior e sendo que as vagas são ocupadas, majoritariamente, por pessoas próximas a meia idade. O que pode afetar o foco de aprendizado deles, devido a rotinas familiares e profissionais.

Nas Figuras a seguir, 6a e 6b, observa-se a opinião sobre a implementação do projeto.
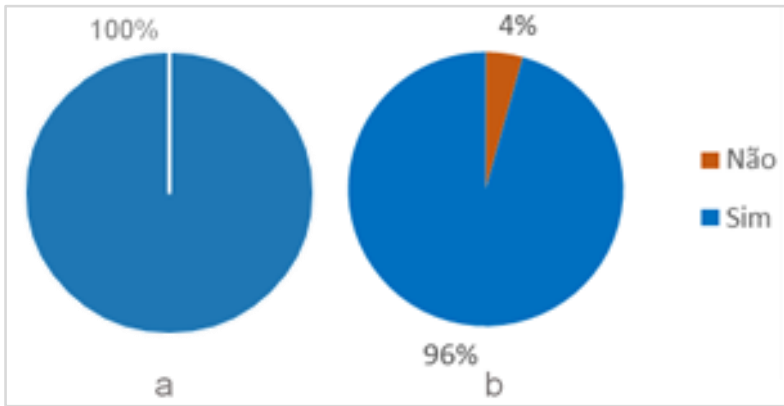

Figura 6 - LabEAD e a implementação na UNIVESP. Fonte: Próprio autor.

A Figura 6a, mostra que $100 \%$ dos entrevistados acreditam ser interessante a implementação de um laboratório remoto na UNIVESP. Já a Figura 6b, mostra que 96\% acreditam que deveria ser empregado em todas as disciplinas que têm conteúdo de laboratório. Esse resultado demonstra a alta necessidade que os alunos têm em relação as práticas laboratoriais durante o ensino do conteúdo teórico. Na Figura 7, pode-se verificar a distribuição dos cursos pesquisados.

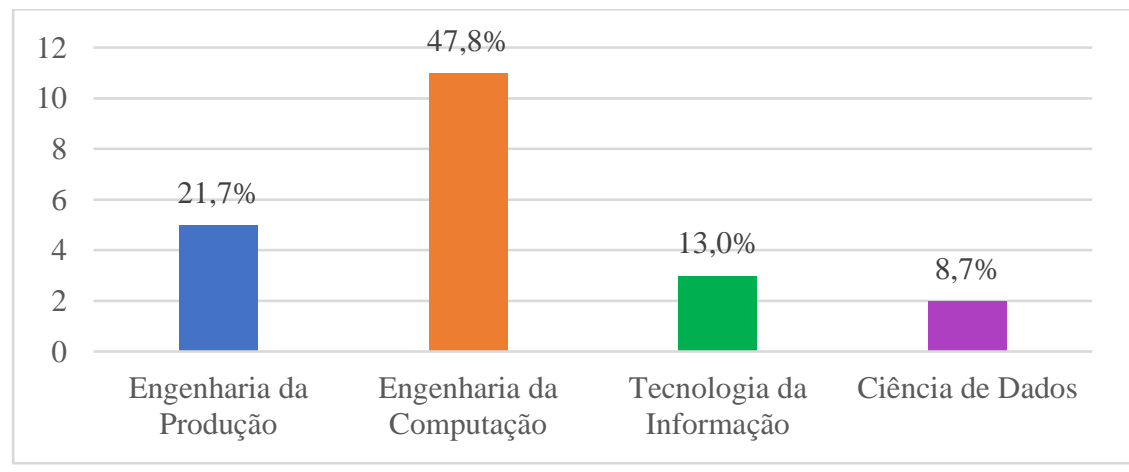

Figura 7 - Cursos dos alunos(as) que participaram da pesquisa. Fonte: Próprio autor.

A Figura 7 apresenta a divisão por cursos dos alunos que participaram da pesquisa. Os alunos de Engenharia da Produção correspondem a 21,7\% dos entrevistados, em azul. A grande maioria dos alunos entrevistados são do curso de Engenharia da Computação, sendo o montante de 47,8\%, em laranja. Os alunos de Tecnologia da Informação (TI) são 
representados em verde, sendo 13,0\% dos entrevistados. E a relação dos alunos de Ciência de Dados é de 8,7\% em roxo. Esses alunos, quando questionados em relação a casos excepcionais, como o COVID-19, responderam que concordam plenamente que as Universidades poderiam utilizar desta ferramenta em substituição temporária do ensino presencial das aulas laboratoriais.

Outro dado importante deste projeto, é que 52\% dos alunos da UNIVESP nunca tiveram contato com um laboratório real como pode-se observar na região azul da Figura 8.

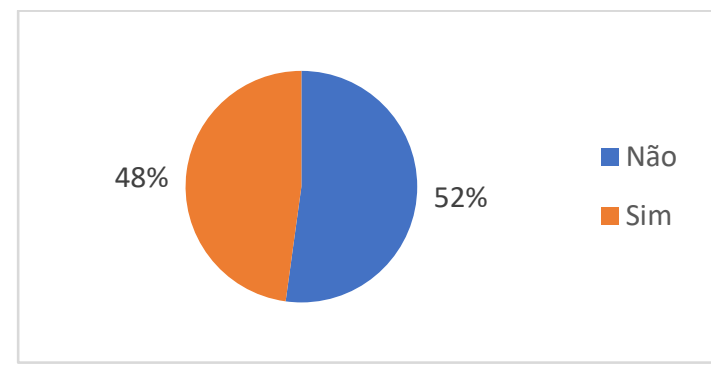

Figura 8 - Contato com laboratório real. Fonte: Próprio autor.

A Figura 9 explora as atribuições dadas pelos alunos na escala likert que vai de 0 a 4 conforme a questão 10 do questionário, sendo 0 quando o aluno discorda totalmente a 4 quando o aluno concorda totalmente. As respostas foram expressas em porcentagem conforme as opiniões dos alunos são demonstradas a seguir.

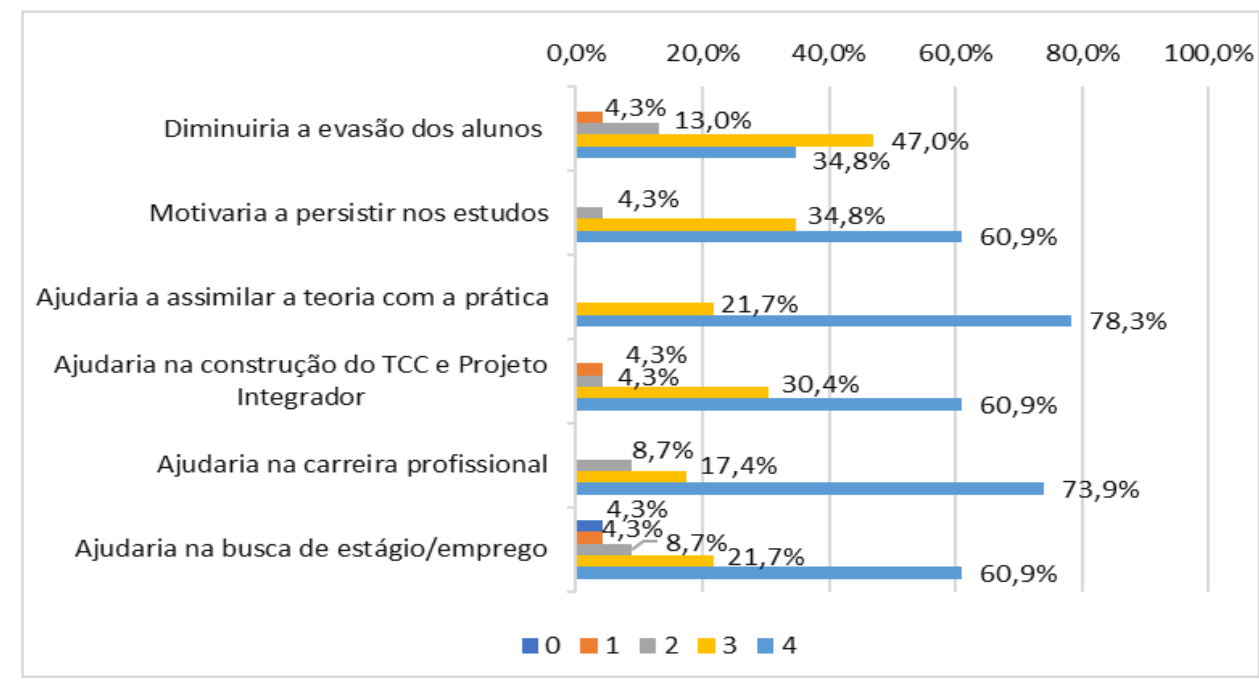

Figura 9 - Atribuições dos alunos na escala likert. Fonte: Próprio autor.

Conforme observado na figura 9, a primeira atribuição mostra que $47 \%$ dos alunos concordam parcialmente que esse projeto diminuiria a evasão escolar, por meio desta informação seria um atrativo aos alunos as aulas, além de uma forma de motivação durante toda a extensão do ensino das disciplinas práticas. Estes dados contribuem com a segunda atribuição, mostrando 60,9\% dos alunos acreditam que esse projeto motivaria a persistir nos estudos, sendo este fator importante no impacto na formação de novos profissionais. Nas demais atribuições, a maioria dos alunos, mais de 60,9\%, acredita que o projeto LabEAD ajudaria a assimilar a teoria com a prática, ajudaria no desenvolvimento do TCC e Projeto Integrador, na carreira profissional e na busca de um estágio e/ou emprego. Diante do exposto pelos dados da figura 9, além das aulas 
conseguirem preparar melhor os profissionais, a implementação do LabEAD seria uma ferramenta que aumentaria a motivação dos alunos, a cerca do conhecimento obtido em aulas teóricas com possibilidade de prática com o LabEAD. Portanto é possível inferir que mais de $61 \%$ dos alunos acreditam que poderiam estudar remotamente as práticas de laboratório durante situações extremas como a SARS-CoV-2 e isso possibilitaria inclusive um ensino híbrido no futuro.

\section{Conclusões e propostas futuras}

Seguindo as conclusões de algumas literaturas, VR / AR irá evoluir de forma constante durante os próximos 10 anos, tornando-se uma tecnologia popular do dia a dia entre a população, incluindo ambientes educacionais. Durante a próxima década, as tecnologias virtuais irão revolucionar a maneira como as pessoas interagem de maneira semelhante à Internet e aos smartphones.

Dessa forma, faz sentido tirar proveito dessas tecnologias para facilitar a aprendizagem, isso significa que os alunos terão experiências na primeira pessoa, e irão tomar decisões e interagir após obter feedback, de forma que o aprendizado será possível após um processo de análise e reflexão.

Portanto, conforme apresentado na seção de resultados, a maioria dos entrevistados, aproximadamente $60 \%$, concordam totalmente que este projeto os ajudaria na graduação e profissionalmente em várias questões. Outra informação importante, é a relação dos alunos com experimentos laboratoriais, sendo que mais da metade, 52\% dos entrevistados, nunca tiveram uma oportunidade de realizar experimentos em laboratórios presenciais. Além disso, a média da nota dada (1 a 5) pelos alunos a este projeto de é de 4,61. Portanto, os pesquisadores deste projeto acreditam que esta ferramenta possa ser empregada pela UNIVESP e/ou por outras universidades que atuam com cursos na modalidade a distância.

\section{Referências}

DEBOER, G. A history of ideas in science education. Teachers College Press, 2019.

GARCIA-ZUBIA, J.; CUADROS, J.; ROMERO, S.; HERNANDEZ-JAYO, U.; ORDUÑA, P.; GUENGA, M.; GONZALEZ-SABATE, L.; GUSTAVSSON, I. Empirical analysis of the use of the VISIR remote lab in teaching analog electronics. IEEE Transactions on Education, v. 60, n. 2, p. 149-156, 2016.

GUIMARÃES, V. S.; OLIVEIRA, C. R. S.; OLIVEIRA, J. A.; REIS, R. M.; OLIVEIRA, I. N.; LOPES, M. S. S. Um Ambiente para as Práticas Laboratoriais de Física: Estudo do Pêndulo Matemático. Revista Brasileira de Informática na Educação, v. 21, n. 2, p. 7891, 2013.

GUTIÉRREZ, M. J.; MORA C.; AÑORBE, D. B; GONZÁLEZ, MARRERO A. Virtual Technologies Trends in Education. Eurasia Journal of Mathematics, Science and Technology Education, v. 13, n. 2, p. 469-486. DOI: https://doi.org/10.12973/eurasia.2017.00626a.

HAYASHI, V.; HAYASHI, F. LabEAD Laboratório Eletrônico de Ensino a Distância durante o Distanciamento Social. V Congresso sobre tecnologias na Educação (Ctrl+E 2020), João Pessoa - Online, agosto de 2020.

HERADIO, R.; TORRE, L.; JAVIER, D. G.; CABRERIZO, E. H.; DORMIDO, S. Virtual and remote labs in education: A bibliometric analysis. Computers \& Education, v. 98, p. 14-38, 2016. DOI: https://doi.org/10.1016/j.compedu.2016.03.010. 
JUNIOR, F. S.; GERMANO, J.S.E.; De Oliveira, J.M.P. (2012) "WEBLAB-Um Ambiente de Laboratórios de Acesso Remoto Educacional." Brazilian Symposium on Computers in Education (Simpósio Brasileiro de Informática na Educação-SBIE). Vol. 23. No. 1.

LICCARDO, A.; ARPAIA,P.; BONAVOLONTÀ, F.; CAPUTO,E. DE PANDI, F.; GALliCCHIO, V.; GLORIA, A.; LO MORIELlO, R. "An Augmented Reality Approach to Remote Controlling Measurement Instruments for Educational Purposes During Pandemic Restrictions," in IEEE Transactions on Instrumentation and Measurement, vol. 70, pp. 1-20, 2021, Art no. 5503420. DOI: 10.1109/TIM.2021.3101314.

LIMA, N.; VIEGAS, C.; ALVES, G.; PEÑALVO, F. J. G. VISIR's usage as an educational resource: a review of the empirical research. In Proceedings of the Fourth International Conference on Technological Ecosystems for Enhancing Multiculturality (TEEM '16). Association for Computing Machinery, New York, NY, USA, 893-901, 2016. DOI: https://doi.org/10.1145/3012430.3012623.

PEREIRA， J.; SIMÃO，J.P.S; DA SILVA，I.N; ALVES， J.B.M; DA SILVA, J.B.;ALVES,G.R. (2018). Implantação e Utilização do Laboratório Remoto VISIR em Instituições de Ensino Técnico, Tecnológico e Superior. Revista Tecnologias na Educação, 1-10.

RACZYŃSKI M. Remote laboratory classes in a COVID pandemic situation. Example of subjects: Microcontroller programming and electronic circuits. The International Journal of Electrical Engineering \& Education. April 2021. DOI:10.1177/00207209211004219.

TORI, R. Educação sem distância: as tecnologias interativas na redução de distâncias em ensino e aprendizagem. Editora: Senac São Paulo; São Paulo, 2010. 